\title{
On a digital approximation for pseudo-differential operators
}

\author{
Alexander Vasilyev and Vladimir Vasilyev* \\ Belgorod National Research University, Studencheskaya 14/1, Belgorod 308007, Russia
}

Copyright line will be provided by the publisher

\section{Introduction}

We introduce a concept of a discrete pseudo-differential operator using general ideas of the theory and would like to show correlations between continuous and discrete cases.

\section{Digital pseudo-differential operators}

\subsection{Digital Fourier transform}

Given function $u_{d}$ of a discrete variable $\tilde{x} \in h \mathbf{Z}^{m}, h>0$, we define its discrete Fourier transform by the series

$$
\left(F_{d} u_{d}\right)(\xi) \equiv \widetilde{u}_{d}(\xi)=\sum_{\tilde{x} \in h \mathbf{Z}^{m}} e^{i \tilde{x} \cdot \xi} u(\tilde{x}) h^{m}, \xi \in \hbar \mathbf{T}^{m}
$$

where $\mathbf{T}^{m}=[-\pi, \pi]^{m}, \hbar=(2 \pi h)^{-1}$, partial sums are taken over cubes

$$
Q_{N}=\left\{\tilde{x} \in \mathbf{Z}^{m}: \tilde{x}=\left(\tilde{x}_{1}, \cdots, \tilde{x}_{m}\right), \max _{1 \leq k \leq m}\left|\tilde{x}_{k}\right| \leq N\right\}
$$

\section{2 $h$-operators and $\hbar$-symbols}

Let $D \subset \mathbf{R}^{m}$ be a domain, and $D_{d}=D \cap h \mathbf{Z}^{m}$.

We consider the following operators

$$
\left(A_{d} u_{d}\right)(\tilde{x})=\int_{\hbar \mathbf{T}^{m}} \sum_{\tilde{y} \in D_{d}} e^{i(\tilde{y}-\tilde{x}) \cdot \xi} \widetilde{A}_{d}(\xi) \tilde{u}_{d}(\xi) d \xi, \tilde{x} \in h D_{d}
$$

and the function $\tilde{A}_{d}(\xi), \xi \in \hbar \mathbf{T}^{m}$ is called a symbol of the operator $A_{d}$.

Also the function

$$
A_{d}(\tilde{x})=\int_{\hbar \mathbf{T}^{m}} e^{i \tilde{x} \cdot \xi} \tilde{A}_{d}(\xi) d \xi
$$

is called a kernel of the operator $A_{d}$.

Definition 2.1 The symbol $\widetilde{A}_{d}(\xi)$ is called an elliptic symbol of the operator $A_{d}$ if ess $\inf _{\xi \in \hbar \mathbf{T}^{m}}\left|\tilde{A}_{d}(\xi)\right|>0$.

Example 2.2 The digital Laplacian is the following

$$
\left(\Delta_{d} u_{d}\right)(\tilde{x})=h^{-2} \sum_{k=1}^{m}\left(u_{d}\left(x_{1}, \cdots, x_{k}+2 h, \cdots, x_{m}\right)-2 u_{d}\left(x_{1}, \cdots, x_{k}+h, \cdots, x_{m}\right)+u_{d}\left(x_{1}, \cdots, x_{k}, \cdots, x_{m}\right)\right),
$$

and its symbol is the function

$$
\tilde{\Delta}_{d}(\xi)=h^{-2} \sum_{k=1}^{m}\left(e^{i h \xi_{k}}-1\right)^{2}
$$

Example 2.3 The digital Calderon-Zygmund operator is defined as follows [4]

$$
\left(K_{d} u_{d}\right)(\tilde{x})=\sum_{\tilde{y} \in h D_{d}} K_{d}(\tilde{x}-\tilde{y}) u_{d}(\tilde{y}) h^{m}, \tilde{y} \in h D_{d},
$$

* Corresponding author: e-mail vbv57@inbox.ru, phone +74 722 301 300, fax +74722 301012 


\section{A comparison between discrete and continual cases}

\subsection{An approximation rate}

Let $P_{h}$ be a projection $\mathbf{R}^{m} \rightarrow \mathbf{Z}^{m}$ so that a function $u$ defined on $\mathbf{R}^{m}$ corresponds to a function $u_{d}$ of a discrete variable defined on $h \mathbf{Z}^{m}, P_{h} u=u_{d}$. If we consider the equation

$$
(A u)(x)=v(x), x \in D,
$$

where $A$ is a classical pseudo-differential operator with the symbol $\tilde{A}(\xi)[1-3]$ of the form

$$
(A u)(x)=\iint_{D} \int_{\mathbf{R}^{m}} e^{i(x-y) \cdot \xi} \tilde{A}(\xi) u(y) d y d \xi,
$$

which acts in certain functional spaces $X \rightarrow Y$, for example Sobolev-Slobodetskii spaces [3]. We say that an element $u \in X$ is an admissible element if $P_{h} u$ is defined.

Definition 3.1 An approximation rate for operators $A$ and $A_{d}$ on an admissible element $u \in X$ is called the following norm

$$
\mu_{h}\left(A, A_{d}, u\right)=\left\|\left(A_{d} P_{h}-P_{h} A\right) u\right\|_{X_{h}},
$$

where $X_{h}$ is so-called digital realization of the space $X$ so that the operator $A_{d}: X_{h} \rightarrow Y_{h}$ is a linear bounded operator.

One of main problems is the following. How we can choose the operator $A_{d}$ to obtain a good approximation rate for the operator $A$ ? We need to fix a domain $D$ and spaces $X, Y$.

Theorem 3.2 Let $D$ be a domain with a Lipschitz, boundary and $X=Y=L_{2}(D), X_{h}=Y_{h}=L_{2}\left(D_{d}\right)$. If $\tilde{A}(\xi)$ is a smooth bounded function on $\mathbf{R}^{m}$ and

$$
A_{d}(\tilde{x})=\int_{\mathbf{R}^{m}} e^{i \tilde{x} \cdot \xi} \tilde{A}(\xi) d \xi
$$

then $\mu_{h}\left(A, A_{d}, u\right) \leq c_{u} h$ for arbitrary smooth function $u \in L_{2}(D), c_{u}$ is a constant.

\subsection{Digital solution and comparison}

Definition 3.3 A digital solution for the equation (2) is called a solution of the equation

$$
\left(A_{d} u_{d}\right)(\tilde{x})=\left(P_{h} v\right)(\tilde{x}), \tilde{x} \in D_{d},
$$

if it exists.

Remark 3.4 It is not evidently that a digital solution always exists. Thus, second of main problems is obtaining a solvability for the equation (3) in the space $X_{h}$ at least for small $h$ from the solvability of the equation (2) in the space $X$. For this purpose we need to study a solvability of discrete equations, some steps in this direction were done in [6,7] for special conical domains $D$ and for the whole space $\mathbf{R}^{m}$ and the half-space $\mathbf{R}_{+}^{m}$ [4].

Theorem 3.5 Let $D$ be $\mathbf{R}^{m}$ or $\mathbf{R}_{+}^{m}$, the conditions of above theorem hold, $A$ be an elliptic invertible operator, $u$ be a solution of the equation (2) with a smooth right-hand side $v, u_{d}$ be a solution of the equation (3). Then

$$
\left\|P_{h} u-u_{d}\right\|_{X_{h}} \leq c h .
$$

\section{Conclusion}

In authors' opinion these considerations will be useful for studying certain applied problems [5] because such operators and equation are very typical for these problems.

Acknowledgements This work has supported by the State contract of the Russian Ministry of Education and Science (contract No $1.7311 .2017 / \mathrm{B})$.

\section{References}

[1] Taylor, M.E.: Pseudodifferential Operators. Princeton Univ. Press, Princeton (1981)

[2] Treves, F.: Introduction to Pseudodifferential Operators and Fourier Integral Operators. Springer, New York (1980)

[3] Eskin, G.: Boundary Value Problems for Elliptic Pseudodifferential Equations. AMS, Providence (1981)

[4] Vasilyev, A.V., Vasilyev, V.B.: Approximation rate and invertibility for some singular integral operators. Proc. Appl. Math. Mech. 13, 373-374 (2013)

[5] Oppenheim, A.V., Schafer, R.W.: Digital Signal Processing. Prentice Hall, Englewood Cliffs, NJ (1975)

[6] Vasilyev, V.B.: Discrete equations and periodic wave factorization. AIP Conf. Proc. 1759, 5 pp. (2016)

[7] Vasilyev, V.B.: Discrete operators in canonical domains. WSEAS Trans. Math. 16, 197-201 (2017) 